\title{
THE THEORETICAL AND EXPERIMENTAL STUDY OF DETERMINISTIC CHAOS PHENOMENA IN A PROCESS OF LIQUID DROPLETS FORMATION
}

\author{
M. R. RZASA \& B. DOBROWOLSKI \\ Department of Mechanical Engineering, Opole University of Technology, Poland.
}

\begin{abstract}
The subject matter of this paper involves the examination of the process of liquid drop formation at the outlet of a nozzle. A theoretical model of the formation of liquid droplet has been developed. The model is based on a recursive equation. Solution of this equation is obtained by the solution set, which is similar to the process of liquid droplets formation. The objective is to demonstrate that a recursive theoretical model can reproduce the chaotic behavior of physical phenomena, which is the formation of liquid droplets. In this paper, it is the assessment of the non-stationary characteristics in the process of drop formation. The potential for describing it in terms of principles familiar from deterministic chaos is presented. The paper contains the results of experiments which indicate that chaotic phenomena occur during the formation of liquid drops. The results of the research are elaborated and presented in the form of attractors and power spectrum diagrams. The ranges of the parameters are determined for which the time intervals between the successive drops tend to be non-stationary. Their presentation in the phase space has revealed the occurrence of chaotic phenomena. It was concluded that numerical calculations and the results gained from experiment are comparable. Consequently, it was indicated that relatively simple mathematical model can be used for simulation of the states of actual physical processes.
\end{abstract}

Keywords: attractors, chaos, drop formation, mathematical models.

\section{INTRODUCTION}

Formation of the liquid drop occurs in many industrial processes such as the condenser and scrubbers. The process of liquid drop formation has an important effect on the duration of the drying process and removal of moisture from gases. Recently, a lot of research has been concerned with condensing water which is included in process gases due to an increase in the efficiency of boilers in which biomass is used. The processes of liquid drop formation in condensing apparatus often display non-stationary characteristics. Therefore, this paper deals with the question of non-stationary characteristics in the process of liquid drop formation and the potential for describing it by the use of principles known from deterministic chaos.

The phenomenon of deterministic chaos has been a familiar one for years. A hundred years ago, Henri Poincare and his team noted that systems consisting of mutually dependent elements can behave in an unpredictable way under certain circumstances. In recent years, this observation was extended to include a number of physical and chemical issues in which chaotic structures are formed from organized structures and vice versa [1,2]. Additionally, a variety of issues are also found in the area of two-phase flows in which deterministic chaos is known to occur [3]. This paper is aimed at the presentation of issues associated with the occurrence of chaos in the process of liquid drop formation. 
The occurrence of chaos in flows was the subject matter of many papers; however, this phenomenon is so complicated that it has not been dealt with sufficiently. Mathematicians from Santa Cruz [4] when conducting research into chaos found its occurrence during the separation of liquid drops. Another paper [5] contains a proposition of a mathematical description of the occurrence of chaos in two-phase flow. The description in it focuses on the application of fractal calculation techniques in the description of dissipative horizontal water flow.

This paper includes results of experimental studies performed by the authors and concerned with the occurrence of chaos phenomenon in the process of liquid drop formation. The experiments involved the measurement of time intervals between the successively separating drops from a nozzle outlet and the subsequent identification of the loss of stability in this process. The results of the measurements are presented in the form of an attractor and calculations of the basic parameters, which describe how the chaos phenomenon is performed. Additionally, the ranges of the value of Reynolds number are identified for which the phenomenon of drop separation has chaotic characteristics. The testing was aimed to confirm the occurrence of deterministic chaos. The methodology was largely based on the fractal calculation techniques.

This paper attempts also to describe the process of the liquid droplets formation with relatively simple recursive equations that allow simulation of chaotic behavior of the droplet formation process.

\section{MATHEMATICAL MODEL}

The model equation that describes the investigated phenomenon is based on the balance of force acting on a drop during its formation on the nozzle outlet (Fig. 1). In accordance with the d'Alembert principle, the equation for the balance of the forces takes the form:

$$
F_{b}=F_{g}-F_{\sigma}
$$

In eqn (1) the particular forces can be expressed in the following way:

gravity force

$$
F_{g}=\frac{4}{3} \cdot \pi \cdot r^{3} \cdot \rho \cdot g
$$

surface tension force

$$
F_{\sigma}=2 \cdot \pi \cdot R \cdot \sigma
$$

inertia force

$$
F_{b}=\frac{4}{3} \cdot \pi \cdot r^{3} \cdot \rho \cdot \frac{d v}{d t}
$$

where $r$ is the instantaneous drop radius $(\mathrm{m}), \rho$ the liquid density $\left(\mathrm{kg} / \mathrm{m}^{3}\right), g$ the gravitational acceleration $\left(\mathrm{m} / \mathrm{s}^{2}\right), R$ the nozzle radius $(\mathrm{m}), \sigma$ the coefficient of surface tension $(\mathrm{N} / \mathrm{m})$ and $v$ the displacement velocity of the specific gravity of a drop $(\mathrm{m} / \mathrm{s})$.

By calculating the volume flow ratio $Q$ as a function of the volume $V$ of a drop, the following formula is derived:

$$
Q=\frac{d V}{d t}=\frac{d\left(\frac{4}{3} \cdot \pi \cdot r^{3}\right)}{d t}=4 \cdot \pi \cdot r^{2} \frac{d r}{d t}=4 \cdot \pi \cdot r^{2} \cdot v
$$






Figure 1: Forces acting on a drop.

After differentiation of $v$ in time, we obtain

$$
\frac{d v}{d t}=\frac{-Q}{2 \cdot \pi \cdot r^{3}} \frac{d r}{d t}
$$

By integrating the above relations into eqn (8), we obtain the following transformed differential equation:

$$
\frac{d r}{d t}=\frac{3 \cdot \pi \cdot R}{\rho \cdot Q} \cdot \sigma-\frac{2 \cdot \pi \cdot \rho \cdot g}{Q} \cdot r^{3}
$$

As a result of transforming eqn (14) into a finite differential form, we obtain a formula which can be used for the iterative calculation of the radius of a separating drop:

$$
r_{i+1}=r_{i}+\frac{3 \cdot \pi \cdot R}{\rho} \cdot M \cdot \sigma-2 \cdot \pi \cdot g \cdot M \cdot r_{i}^{3}
$$

where $M=\Delta t / Q$.

The simulation of chaos involves adapting the value of parameter $M$ in such a way that eqn (8) does not any longer keep a singular, stable solution. The results of numerical calculations are presented in Fig. 2. For low values of parameter $M$ (Fig. 2a) we obtain a stable solution of eqn (8). This state corresponds to the formation of drops without the chaos. Figure $2 b$ illustrates the beginning of the loss of stability in solving this equation. During the initial phase of the iteration process, it is possible to note oscillations, and after that the solution is stationary. Figure $2 \mathrm{c}$ shows the oscillations increase until a stable generation of two solutions is achieved. This represents a case when typical for chaos bifurcations start to appear. After a small increase in the value of parameter $M$, we have to do with multiplication of the number of solutions (Fig. 2d). The further increase in the value of parameter $M$ results in an abrupt increase in the number of solutions of eqn (8). 
a)



b)

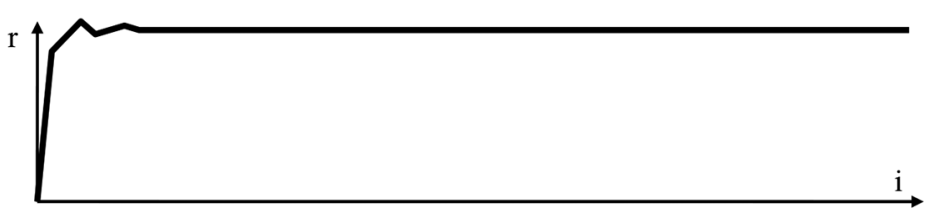

c)

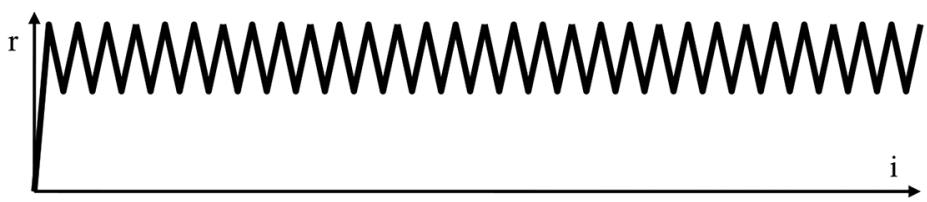

d)

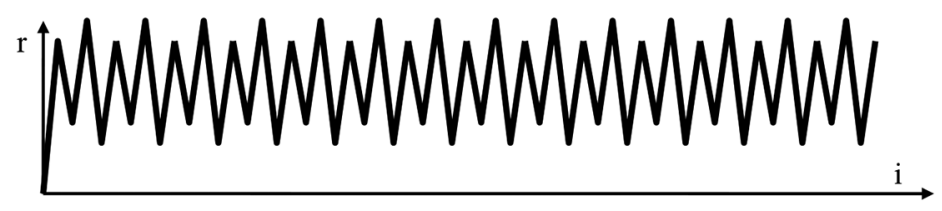

e)

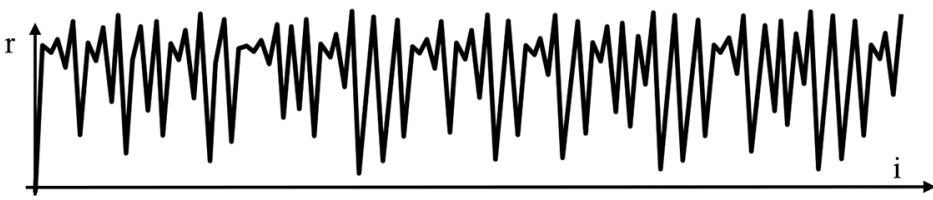

Figure 2: Results of numerical calculation of the radius of the separating drop for the value of parameter M: (a) $M=0.1$, (b) $M=1$, (c) $M=1.4$, (d) $\mathrm{M}=1.6$ and (e) $\mathrm{M}=1.8$

\section{EXPERIMENTAL SETUP}

Several series of experiments were undertaken with the aim of measuring the time intervals between the successive water drops separating from a nozzle outlet. The diagram of the experimental setup is presented in Fig. 3. A tank 1 was installed in a way which ensured free liquid flow along a $\phi 30-\mathrm{mm}$ pipe. Inside the tank is installed a temperature sensor to measure the liquid temperature. A regulation valve 2 was installed at the end of the pipe and was used for the control of the flow rate of the liquid out of the tank. The nozzle at which liquid drops are formed was installed at the end of the pipe. The nozzle was made of brass. At a certain distance from the nozzle, a photocell was installed in order to register the successive instances of liquid drop separation and it was coupled with a computer measuring system. The measurement of flow rate was performed by means of the gravimetric method by the use of measuring the mass of tank 3 .

The value of the liquid mass flow rate $m$ was derived from the relation:

$$
m=\frac{\Delta m}{\Delta t}
$$




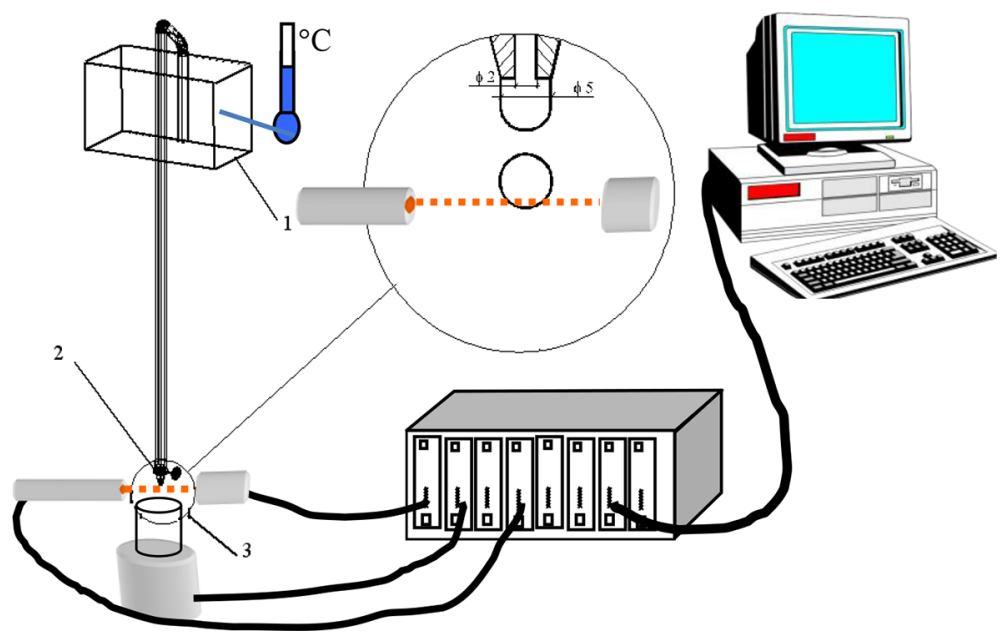

Figure 3: Experimental setup.

where $\Delta m$ is the mass of liquid accumulated in the tank during measurement $(\mathrm{kg})$, and $\Delta t$ the duration of measurement (s).

Following series of measurements were undertaken for various values of the liquid flow rate. Sequence of intervals between the separating drops was measured for a given value of the liquid flow rate (for 3000-5000 drops). Subsequently, the liquid flow rate was increased and the measurements were repeated. Thus, data series for various values of the liquid flow rate were obtained from the testing.

\section{EXPERIMENTAL RESULTS}

An analysis of the successive expressions in series of time intervals between separating liquid drops was undertaken in respect to selected values of the flow rate (Fig. 4). The vertical axis indicates the mean time taken by the successive drops to form. The horizontal axis marks the successive numbers of drops. In order to clearly illustrate the time change, the discreet points marking time intervals are joined by sections. Along with an increase in liquid flow rate, the time taken for the liquid drops to form increases. Also, the irregularity of the drop formation increases as well. In Fig. 4, the process of drop formation as a function of Reynolds number is shown. The Reynolds numbers were derived from the relation:

$$
\operatorname{Re}==\frac{4 \cdot m}{\pi \cdot D \cdot \eta}
$$

where $\eta$ is the liquid viscosity $(\mathrm{Pa} \cdot \mathrm{s})$ and $D$ the nozzle diameter $(D=0.005 \mathrm{~m})$,

The analysis of time series in which liquid drops are formed for an increasing liquid stream discharge from a nozzle is used to capture the mechanism behind the origin of chaos. Figure $4 \mathrm{a}$ and $\mathrm{b}$ presents a series of the time intervals between the drops for a flow rate in which chaos does not yet occur. All of the values are contained close to the middle value. An increase in the liquid flow rate results in an increase in the intensity in which drops separate while a constant level of interference is maintained. Figure $4 \mathrm{c}$ and d presents critical courses for which a transfer is made from a stationary structure to a chaotic one. The formation of regular 

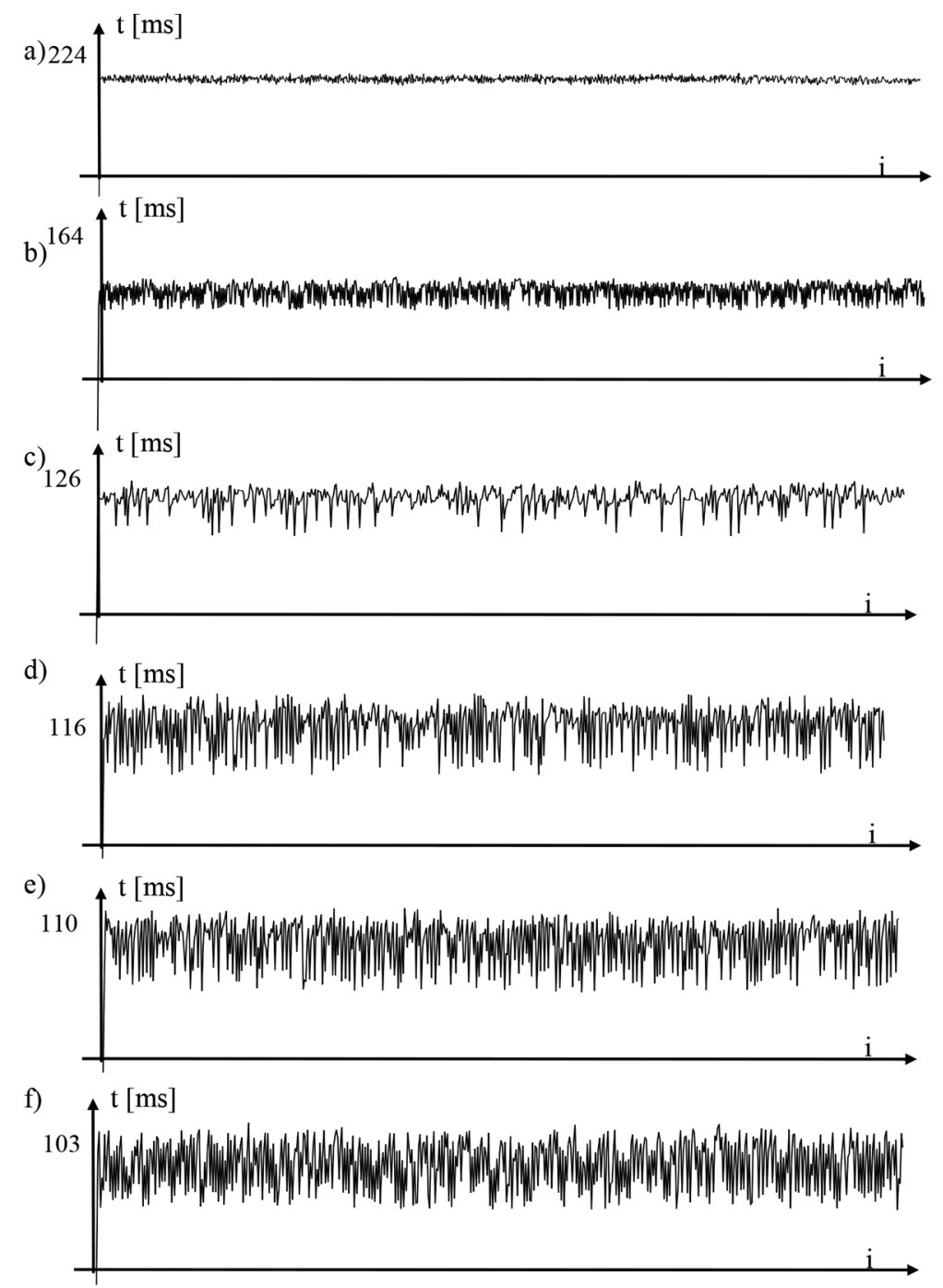

Figure 4: Values of time intervals, $t$ (ms), between the forming drops for: (a) $\operatorname{Re}=109.1$, (b) $\mathrm{Re}=162.1$, (c) $\mathrm{Re}=203.5$, (d) $\mathrm{Re}=213.3$, (e) $\mathrm{Re}==221.7$ and (f) $\mathrm{Re}=226.1$.

drops is discernible and the times of successive drops are considerably different from the mean value. Additionally, their separation times are considerably irregular. According to chaos theory, these represent bifurcation points.

The following charts (in Fig. 4e and f) present deterministic chaos. On the basis of the course of the expressions in the series, it is difficult to note any regularity. Nevertheless, this does not need to mean loss of regularity.

Deterministic chaos is characterized by the occurrence of specific dominating values. However, by merely observing time series it is difficult to note any values which are dominant. On the basis of such observations, it is, however, possible to conclude whether there are dominant values in a given series, which could potentially indicate the occurrence of a deter- 

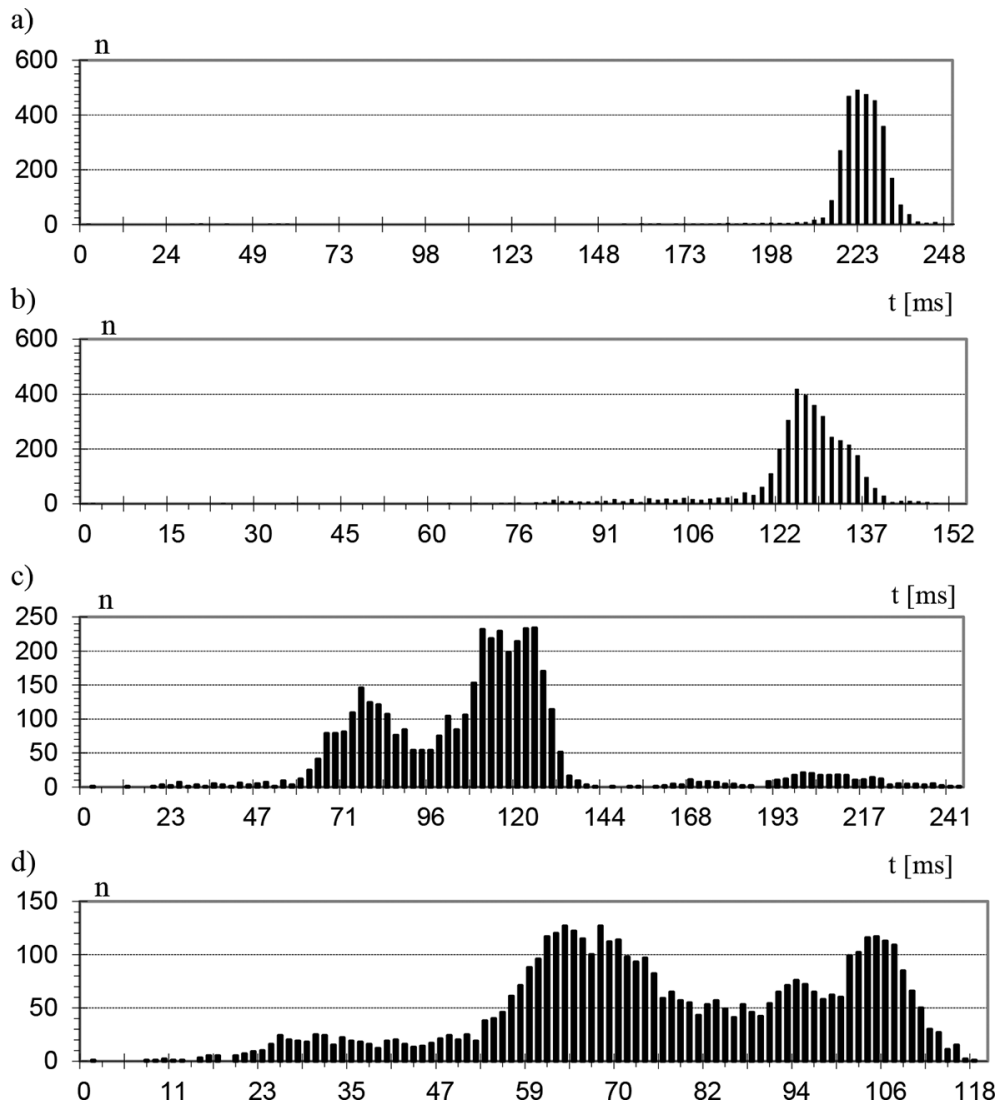

Figure 5: Power spectrum for (a) $\mathrm{Re}=109.1$, (b) $\mathrm{Re}=203.5$, (c) $\mathrm{Re}=226.1$ and (d) $\mathrm{Re}=258.5$.

mined process [4]. The analysis of power spectrum was based on a discreet Fourier transform [6]. On its basis, it was possible to analyze the distribution of numerical values. The range of the values in a series was divided into 100 equal parts and subsequently it was possible to determine how many samples in a given set are contained in each set.

Figure 5 presents the power spectrum for selected series of samples corresponding to the particular values of the Reynolds number. In these charts, $n$ denotes the number of samples in a given time interval $t$.

Figure 5a presents the distribution of a power spectrum for a stable process of liquid drop formation. The distribution of drop formation times of drops is similar to a Gaussian curve. One can therefore conclude that the discrepancies between the measured times are due to the measurement errors. The power spectrum for the flow in the vicinity of the point of stability loss is presented in Fig. 5b. It is very similar to the distribution for stationary flows, however, asymmetry of the power distribution is observed. For the flow from Fig. 5c and d (for which the deterministic chaos is developed), the power spectrum contains a few dominant value ranges. This forms evidence in favor of a statement about the process having multidimensional parameters. Along with the increase in the liquid flow rate, more power bands are formed, which consequently leads to a complete instability of the system and origin of chaos in which none of the process parameters is a dominant one. The above power spectra do not 
yield the values of the time intervals between the forming drops, which are the most probable for a given flow rate. The occurrence of the dominant times of drop formation therefore indicates the presence of deterministic chaos. As a consequence, it is justified to seek for theoretical models, which could generate values that are similar to the ones measured during the experiment.

\section{METHODS OF CHAOTIC DATA COMPARE}

Direct comparison of time series from two non-stationary processes is relatively difficult. One of the methods which allows to see the results of the comparison is the data presentation in field of phase diagrams in which images are presented in the form of attractors. These types of attractors could be described in terms of dimensions used to identify the parameters of fractals [5]. Chaos can be unpredictable, which does not, however, mean that is it not deterministic. This means that two identical chaotic systems which are excited by a single signal behave in an identical way; nevertheless, it is not possible to predict the state of the system at a particular instant in time. By investigating the behavior of complex systems, Tambe and Kulkrnt showed [1] when the influence force between mutually dependent elements of a system increases and the system undergoes a phase shift from an stationary state into a chaotic one. The case is similar with drops. There is no possibility to predict the time interval between the successive drops, although it is possible to determine the set of values in which it will be found.

An attractor was applied in order to present the data in three-dimensional state space. An attractor is a quantity which is dependent on the state of the observed system and characterizes a given phenomenon. This technique involves reconstruction of the shape of an attractor on the basis of a series of measurements. Such reconstruction has already been the subject of study by David Ruelle and Norman Packard [4] and indicates the correctness of the above approach. The results of experimental measurements take the form of a time series. It is a series of numbers which represent the value of an observed quantity performed at constant time intervals. A phenomenon that is observed in this manner is, however, a multidimensional one. Conversely, an attractor should represent the dimensions of a phenomenon and, therefore, it needs to be multidimensional despite the fact that the observations concern only a single quantity. Due to the possibility of presenting results in three dimensions only, the dimensions of an attractor has to be limited to such a number of dimensions. In this manner, three-dimensional time series are obtained on the basis of a series of an observation involving only a single dimension, in which each point has three coordinates $\left(x_{n} ; x_{n-1} ; x_{n-2}\right)$. The line in space encircling these points constitutes the typological approximation of the shape of the attractor.

The measurement of non-linear properties of a dynamic system is aimed at reconstruction of the properties of an attractor on the basis of a registered signal. For this purpose, a phase image is developed on the basis of measurement data for signal $x(t)$, which involves the presentation of data in a $d$-dimensional space (called immersion space). The measurement data form a time series:

$$
\left\{x_{n}\right\}=\left\{x_{1}, x_{2}, \ldots, x_{n}\right\}
$$

A curve $\xi d(t)$ is constructed for an arbitrary immersion dimension $d$ on the basis of experimental data:

$$
\xi_{\mathrm{d}}(t)=\{x(t), x(t+\tau), x(t=2 \tau), \ldots ., x(t+(d-1) \tau)\}=\left\{x_{1}, x_{2}, \ldots . ., x_{n-d-1}\right\}
$$



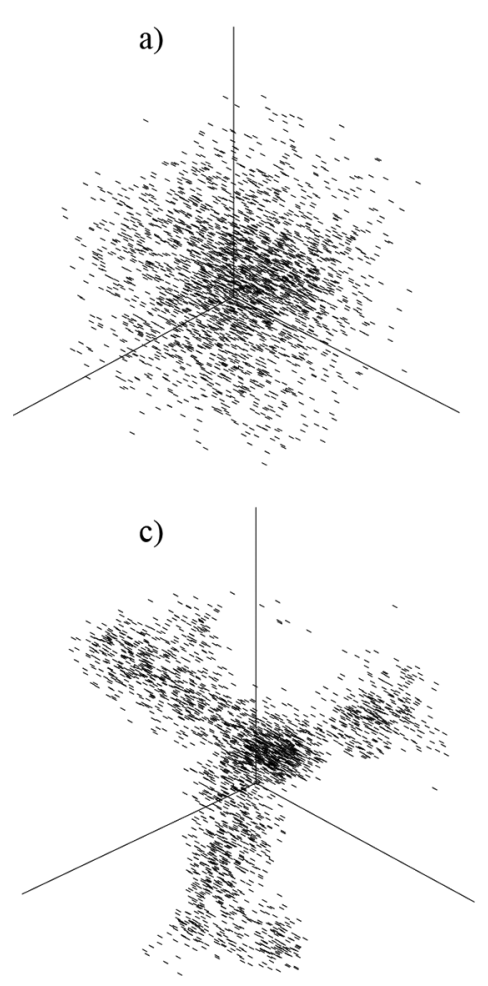


Figure 6: Attractors for (a) $\operatorname{Re}=109.1$, (b) $\operatorname{Re}=203.5$, (c) $\operatorname{Re}==221.7$ and (d) $\operatorname{Re}=226.1$.

During the measurement of time intervals between the drops, it is possible to register accidental and false values (i.e. ones that are dissimilar from the remaining ones), which result from the occurrence of random interference (vibration, noise). In connection with this, $5 \%$ of data with the highest and lowest values have been rejected from consideration in each series.

The scatter of the values of time intervals between the separating drops is contained a certain range. The values of the time scatter are dependent on the velocity of the liquid discharge. The shape of the attractor is not relative to the constant component but only from the time differences between the successive separating drops. Therefore, the constant component was deduced from the resulting numerical values and, consequently, the data were reduced to numbers in the range $0-1000$, in accordance with the formula:

$$
t(i)=\frac{t(i)-t_{\min }}{t_{\max }-t_{\min }} \cdot 1000
$$

where $t(i)$ is the time interval between successive drops, $t_{\max }$ the maximum value of the interval, and $t_{\min }$ the minimum value of the interval.

The attractors for the selected flow rates of the discharged water are presented in Fig. 6. The shapes of the attractors gained from the experiments assume regular fractal forms. Figure $6 \mathrm{a}$ presents an attractor for the liquid flow for which chaos does not already occur. It indicates lack of any order and the completely blurred image indicates that no interference is present in 


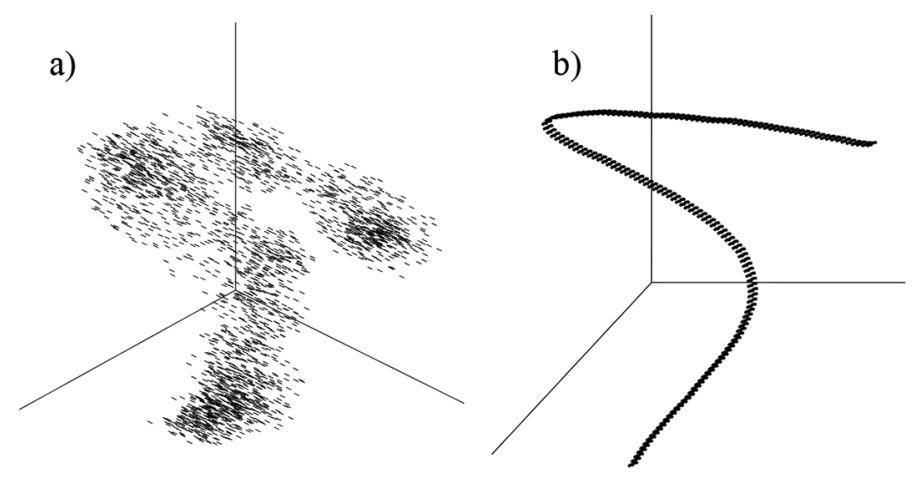

Figure 7: Attractors for a series of (a) experiment data for $\mathrm{Re}=226.1$ and (b) numerical calculations for $M=1.8$.

the form of a white noise. Figure $6 \mathrm{~b}$ presents attractors in the vicinity of a critical point, that is, an attractor which corresponds to the flow rate of liquid from a nozzle for which the structure is in transit state from a stationary into chaotic one. Figure $6 \mathrm{c}$ presents the development of chaos. Some form of regularity is discernible, which means deterministic chaos in the system. Figure $6 \mathrm{~d}$ presents an attractor, which represents a typical form for chaotic behavior. The set of point in the space forms a regular shape for a highly irregular behavior of the time course. The occurrence of chaos in the process of liquid drop formation results from the fact that the chaotic behavior forms a set of regular behaviors, while none of them play a dominant role.

Figure 7 shows the results of the unambiguous similarity between the behavior of the theoretical model and the experiment for a series of the solutions to eqn (8) for $M=1800$, an attractor was generated. In its shape, this attractor is very similar to the ones gained from the experiment as presented in Fig. 7a. The similarity of the attractors in accordance with the chaos theory shows that the behaviors of the systems are similar. This confirms the validity of the assumptions adopted in the theoretical model. The developed mathematical model well represents the parameters of the examined physical phenomenon.

\section{CONCLUSIONS}

The analysis presented in this paper indicates the occurrence of several fundamental characteristics of deterministic chaos in the process of liquid drop formation. It was observed that after exceeding a certain value of liquid flow rate, the process of drop formation changes from regular to a chaotic one. After a critical point is exceeded, the power spectrum changes from individual bands into broadband, which forms a characteristic feature of chaos. On the basis of the observation of this phenomenon, a simple mathematical model was developed, which also can be used to reveal chaotic solutions. In this way, it has been proved that even simple mathematical model can be applied for the simulation of chaotic behavior of real-life processes.

\section{REFERENCES}

[1] Tambe, S.S. \& Kulkrnt, B.D., Intermittency route to chaos in a periodically forced model reaction system. Chem. Eng. Sci. 48(15), pp. 2817-2821, 1993. doi: http:// dx.doi.org/10.1016/0009-2509(93)80193-T 
[2] Van Den Bleek, C.M. \& Schouten, J.C., Can deterministic chaos create order in fluidized-bed scale-up. Chem. Eng. Sci. 48(13), 1993. doi: http://dx.doi.org/10.1016/00092509(93)81058-4

[3] Lacy, C.E., Sheintuch, M. \& Dukler, A.E., Methods of deterministic chaos applied to the flow of thin wavy films. AIChE J. 37(4), pp. s.481-s.489, 1991.

[4] Crutchfield, J.P., Farmer, J.D., Packard, N.H. \& Shaw, R.S., Chaos. Sci. Am. pp. 46-57, 1986.

[5] Franca, F., Acikgoz, M., Lahey, R.T. \& Clausse, A., The use of fractal techniques for flow regime identification. Multiphase Flow, 17(4), 1991.

[6] Bracewell, R.N., The Fourier Transform and Its Applications, 3rd edn., McGraw-Hill Book Company: New York, 2000. 